\title{
Evolution dans le temps de l'envasement dans une retenue de barrage dans lequel est pratiquée la technique du soutirage
}

\author{
par B. Remini \\ Institut de Génie rural, Université de Blida (Algérie) \\ J.-M. Avenard \\ (UFR de Géographie, ULP de Strasbourg) \\ A. Kettab \\ ENP Alger (Algérie)
}

\begin{abstract}
Du fait de la forte accélération de l'envasement qui menace les barrages algériens, les services concernés utilisent la technique du soutirage, définie comme étant une méthode pratique qui consiste à évacuer les sédiments par des pertuis de vidange (vannes et vannettes de dévasement) dans le but de réduire au minimum l'accumulation des apports.

L'utilisation de cette technique doit cependant être différente selon le barrage considéré, et plus précisément selon les conditions qui président à son exploitation : importance des apports de sédiments, état de marche des systèmes de vidange, modifications apportées à la digue etc.

Il en résulte que la détermination d'une loi d'évolution dans le temps des dépôts des sédiments dans une retenue de barrage, dans lequel cette technique est pratiquée, est indispensable pour :

- la précision de l'envasement de la retenue,

- l'évaluation de l'efficacité de la technique du soutirage,

- l'évaluation de la durée de vie de l'ouvrage.

Quatre facteurs peuvent ainsi être retenus, qui vont déterminer l'évolution dans le temps de l'envasement dans une retenue de barrage :

- un faible taux de comblement,

- une vanne de fond complètement obturée,

- un taux de comblement important,

- une surélévation de la digue du barrage.
\end{abstract}

\section{I $\square$ FAIBLE TAUX DE COMBLEMENT}

Durant les premiers temps de l'exploitation du barrage, les sédiments se déposent de manière uniforme sur toute la surface de la retenue. La pratique de la technique de soutirage est peu efficace, et il y aura peu de sédiments évacués pour une proportion d'eau importante à cause de la hauteur des seuils des vannes par rapport au niveau du fond de la retenue (fig.1) :

- même si les pertuis de vidange sont ouverts au moment opportun, une faible quantité de vase sera évacuée, laissant un volume important de particules se décanter ;

- si les pertuis restent fermés, toute la masse solide se déposera au fond de la cuvette, c'est-à-dire que toutes les particules entrées dans la retenue sont piégées : il en résulte une évolution linéaire de l'envasement en fonction du temps.

C'est effectivement ce que montre l'étude des barrages de Cheffia, Sarno et Mefrouch dont les taux de comblement sont faibles. Les résultats obtenus, représentés sur la figure 2 $(\mathrm{a}, \mathrm{b}, \mathrm{c})$, indiquent clairement que l'évolution dans le temps de l'envasement est linéaire, ce qui signifie que le toit de la vase est parallèle au fond de la retenue.

\section{II $\square$ VANNE DE FOND COMPLÈTEMENT OBTURÉE}

Nous avons la chance, pour l'étude de cette situation, d'avoir l'exemple du barrage de l'Oued El Fodda, durant la période d'obturation de la vanne de fond (1948-1961), période pendant laquelle aucune perte en sédiments n'a été enregistrée. En effet, la vanne avait été complètement obturée en 1948 à la suite de dépôts de vase, et les cinq pertuis de dévasement, placés dans le corps du barrage pour son remplacement, n'ont été mis en service que durant l'année 1961.

La figure 3, représentant l'évolution dans le temps de l'envasement dans la retenue durant cette période, montre qu'ici encore la fonction est une droite linéaire.

\section{Evolution in time of the sedimentation in a reservoir of dam in which is practised the technical of the drawing off}

The evolution of deposits to the sediments (drained by the density currents) in the reservoirs of dams in Algeria is approached by four initial situations : feeble or strong rate of the filling, gate of the bottom obturated and raising of dyke. The results obtained show that the drawing off to the solid particles by the use of the sluices of draining influence on the progress of the sedimentation in the basin. Into action, in the beginning of the exploitation of dam, the sedimentation perform linearly with time, but as soon as the roof of mud reaches gates threshold, the function of sedimentation takes a polynomial form of second degree. 


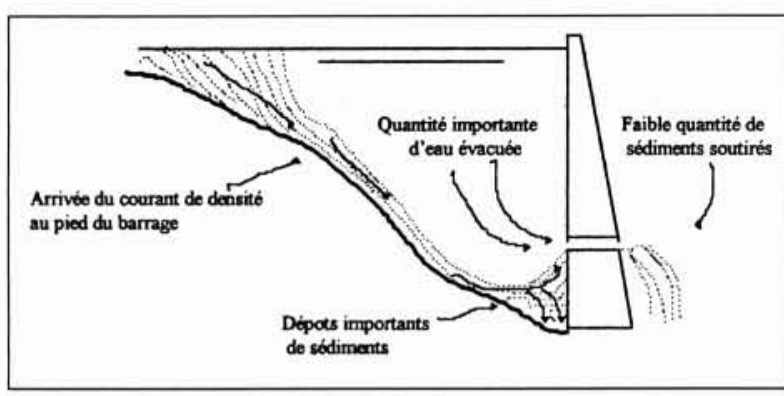

1. Soutirage des sédiments dans un barrage nouvellement mis en exploitation.

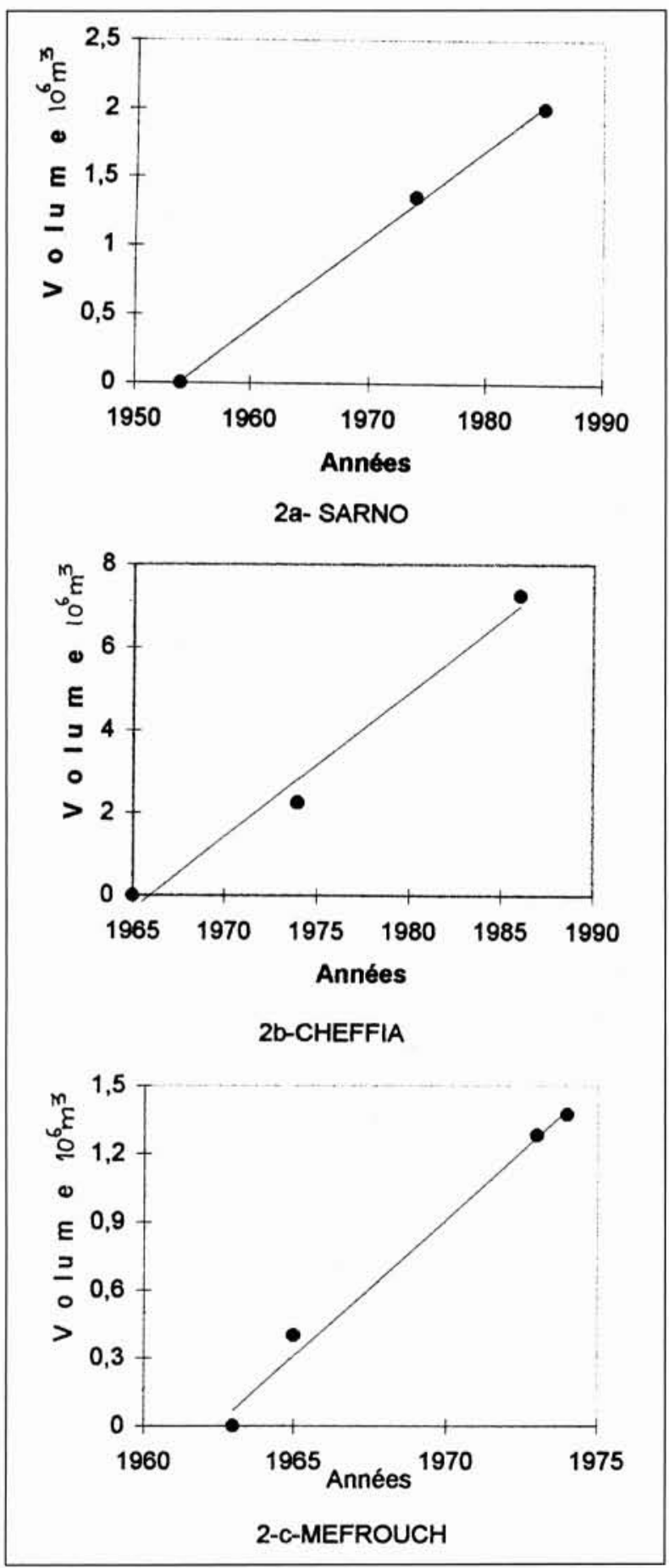

2. Evolution dans le temps de l'envasement dans des retenues de barrage à faible taux de comblement.

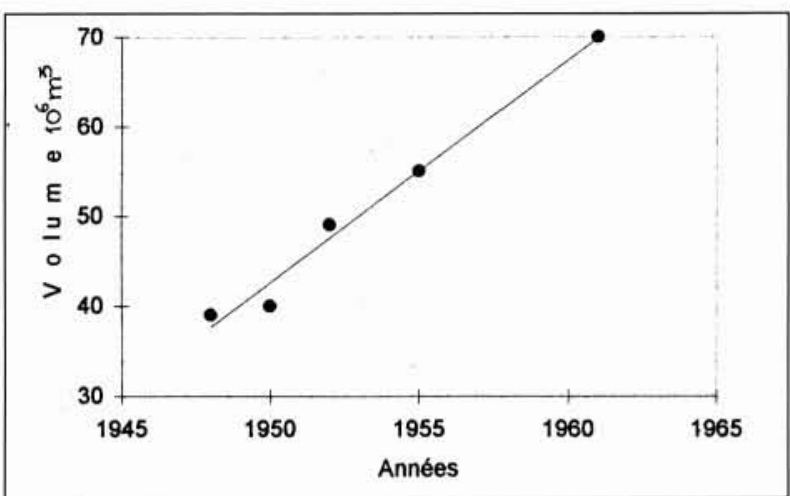

3. Evolution dans le temps de l'envasement dans la retenue du barrage de l'OUED EL FODDA durant la période de blocage de la vanne de fond (1948-1961).

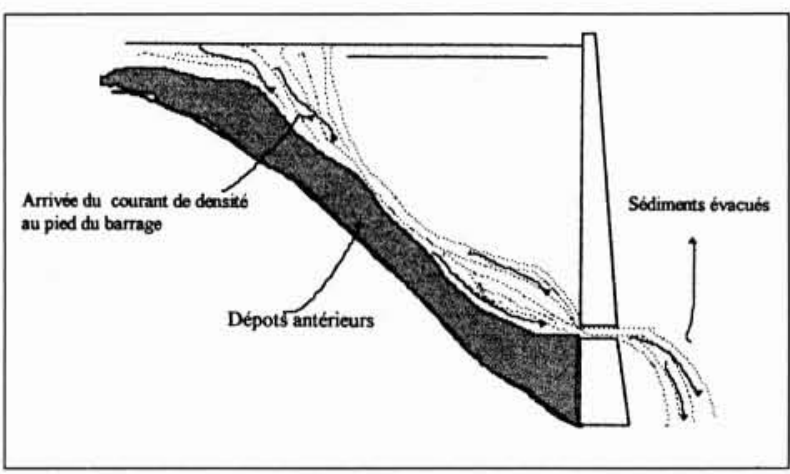

4. Soutirage des sédiments dans un barrage à fort taux de comblement.

\section{III — TAUX DE COMBLEMENT IMPORTANT}

Dès que le toit de la vase atteint le seuil des vannes, la couche turbide se trouve au dessus, et son soutirage devient plus facile (fig.4) ; en conséquence une quantité importante de sédiments sera évacuée, et la vitesse de sédimentation sera réduite par rapport aux premières années de l'exploitation : ce ralentissement de l'envasement se traduira donc par une évolution dans le temps qui ne sera plus linéaire mais polynomiale du deuxième degré, avec même une tendance à la stabilisation.

La figure 5 (a à f), représente l'évolution dans le temps dans les retenues des barrages d'Ighil Emda, Oued El Fodda, Ghrib, Foum El Gherza, Beni Bahdels et Bouhanifia, utilisant habituellement la technique du soutirage, et qui ont enregistré un taux de comblement élevé : il est possible de constater que la fonction est effectivement polynomiale du deuxième degré, et liée à une nette régression de la vitesse de sédimentation dans le temps.

\section{IV — SURÉLÉVATION DE LA DIGUE D'UN BAR- RAGE}

Pour compléter cette approche, le cas d'un barrage dont la digue a été surélevée peut paraître intéressant, et nous avons à cet effet représenté sur la figure 6 (a et d) l'évolution dans le temps de l'envasement dans les retenues de Zardezas, K'Sob, Boughezoul et Bakhada dont les digues ont été respectivement surélevées en 1975, 1977, 1960 et 1958 à la suite d'un comblement rapide des réservoirs.

On peut constater que :

- le graphe est composé de deux courbes, la première correspondant à une évolution de la sédimentation dans la retenue 


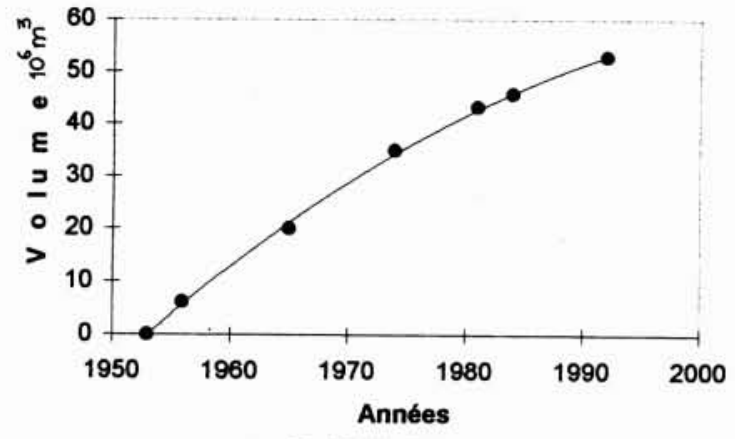

5a-IGHIL EMDA

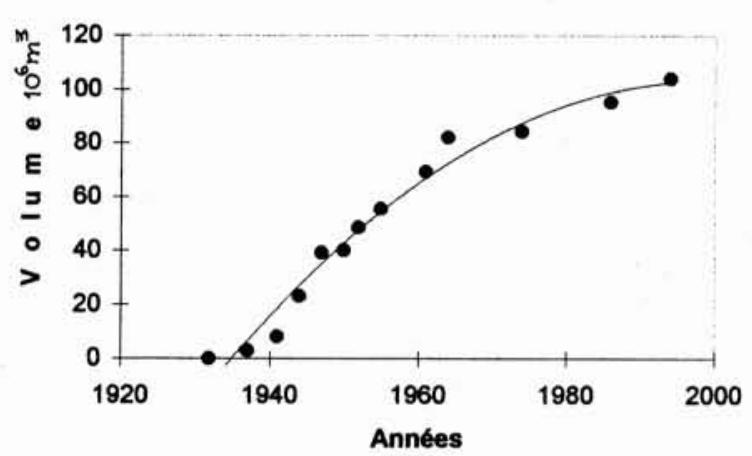

5b-OUED EL FODDA

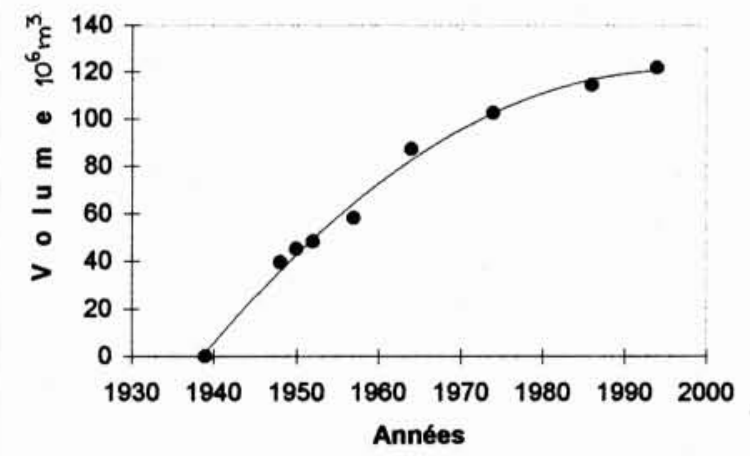

5 c- GHRIB

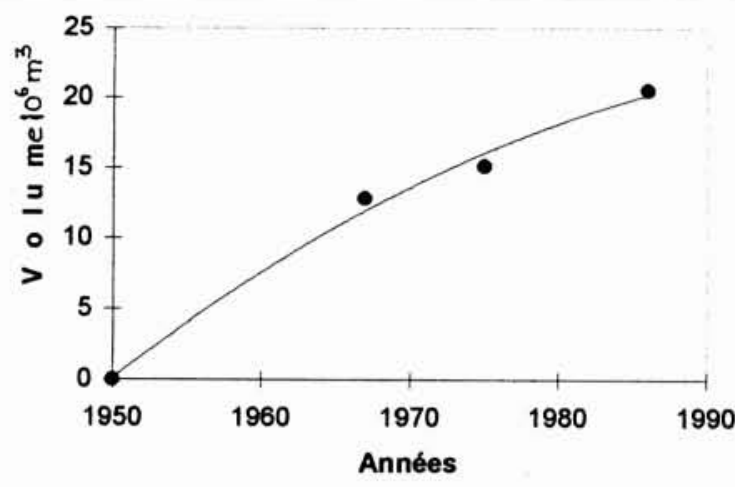

5d-FOUM EL GHERZA

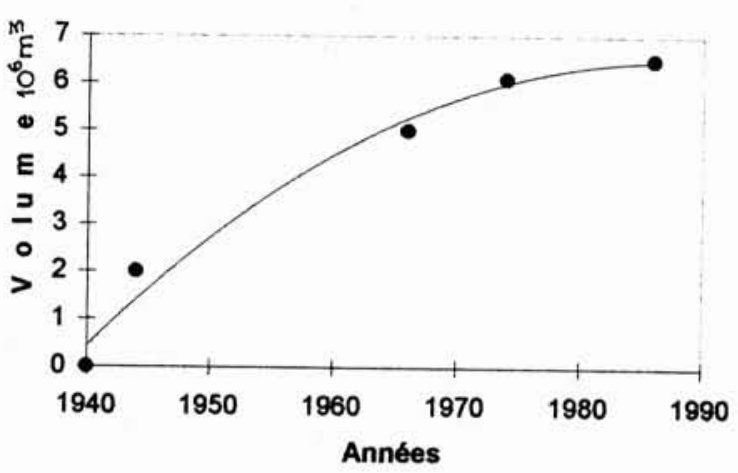

5 e- BENI BAHDELS

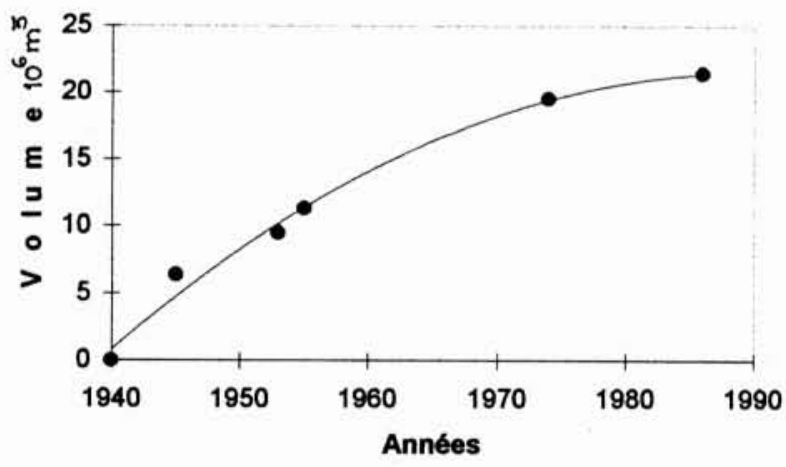

$5 \mathrm{f}$ - BOUHANIFIA

5. Evolution dans le temps de l'envasement dans les retenues à fort taux de comblement.

avant la surélévation de l'ouvrage, la deuxième à cette même évolution après surélévation ;

- l'envasement augmente plus rapidement après la surélévation. Les vitesses moyennes de sédimentation dans les deux retenues sont en effet nettement supérieures après la surélévation comme indiqué dans le tableau 1.

Ceci tendrait donc à prouver qu'au début de l'exploitation d'une réserve d'un barrage, l'envasement serait beaucoup plus important qu'après.

A notre avis, le ralentissement dans le dépôt des sédiments dans le temps n'est pas uniquement dû à l'efficacité du soutirage, mais aussi au profil en long du toit de vase qui évolue vers un profil d'équilibre. Pour donner du poids à cette idée, nous avons représenté sur la figure 7 ( $\mathrm{a}$ et b) l'évolution dans le temps de l'envasement dans les retenues de barrages

\begin{tabular}{|l|c|c|}
\hline \multirow{2}{*}{ Barrage } & \multicolumn{2}{|c|}{ Vitesse de sédimentation $10^{6} \mathrm{~m}^{3} /$ an } \\
\cline { 2 - 3 } & Avant surélévation & Après surélévation \\
\hline ZARDEZAS* & 0,30 & 0,70 \\
\hline K'SOB & 0,25 & 0,29 \\
\hline BOUGHZOUL & 0,34 & 0,50 \\
\hline BAKHADA & 0,05 & 0,15 \\
\hline
\end{tabular}

*Les pertuis de soutirage ont été obturés en 1984.

d'Ighil Emda et Oued El Fodda, en supposant qu'aucun soutirage n'ait été effectué : nous constatons que la fonction reste toujours polynomiale du deuxième ordre. 


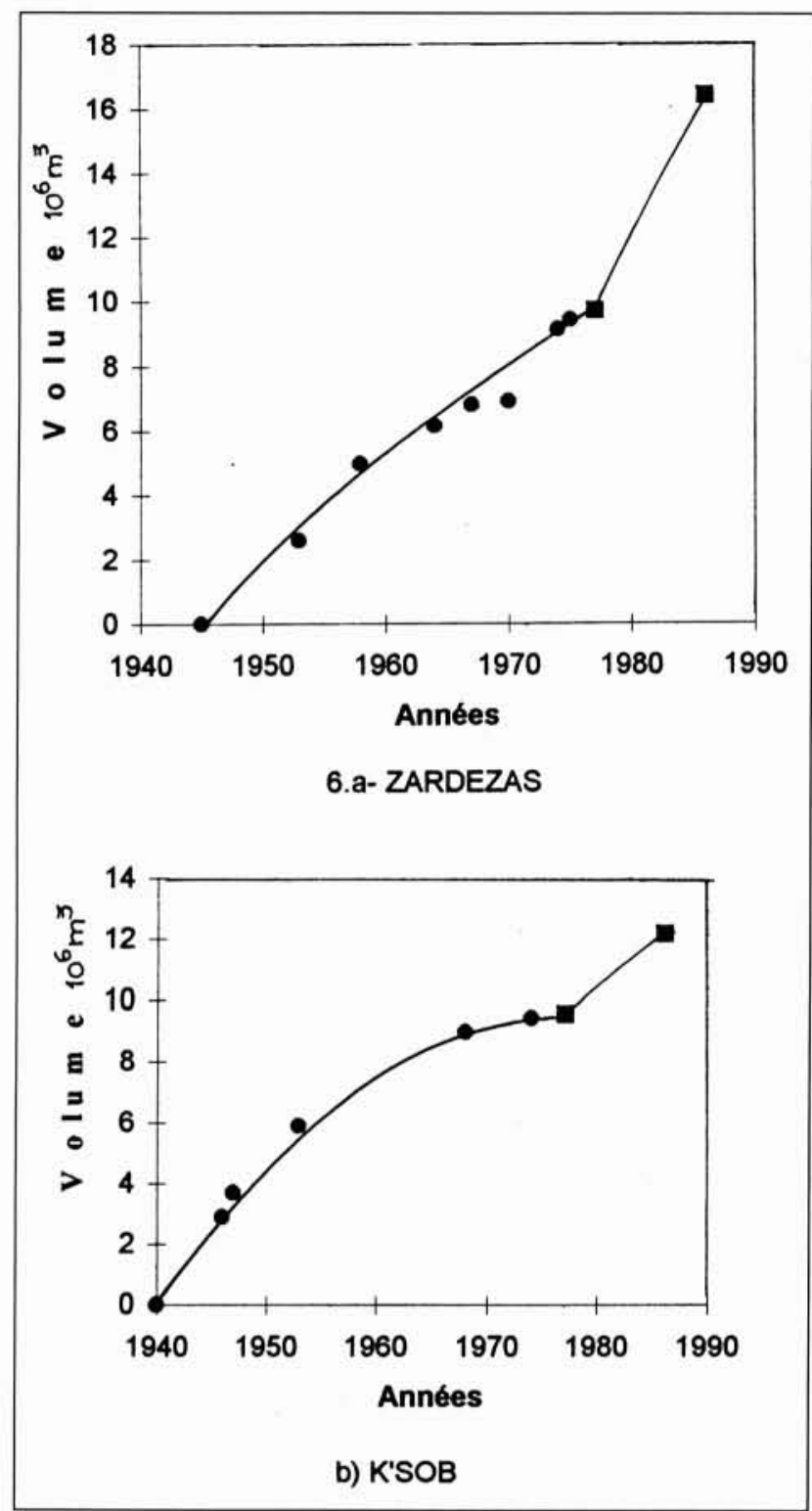

6. Evolution dans le temps de l'envasement dans les retenues de barrages surélevés.

\section{V $\square$ CONCLUSION}

L'utilisation courante des vannes de fond d'un barrage comme moyen de réduction de l'envasement influe directement sur l'évolution du profil des dépôts de vase dans la cuvette. En effet, au début de l'exploitation d'un barrage, le remplissage de la retenue par les sédiments suit une loi linéaire en fonction du temps, et dès que le toit de la vase atteint le seuil des vannes, la loi devient polynomiale (du deuxième degré). Ce ralentissement n'est pas dû uniquement au soutirage, mais le profil en long tend vers un profil d'équilibre.

\section{Références}

[1] Chadi A., HADI T. - 1992- Erosion des sols et envasement des barrages. Revue Algérie Equipement $\mathrm{N}^{\circ} 5$, juillet.

[2] Drouhin et al -1951- La sédimentation des barrages réservoirs en Algérie. Quatrième congrès des grands barrages, question $\mathrm{N}^{\circ} 14$, New Delhi, 163-195.

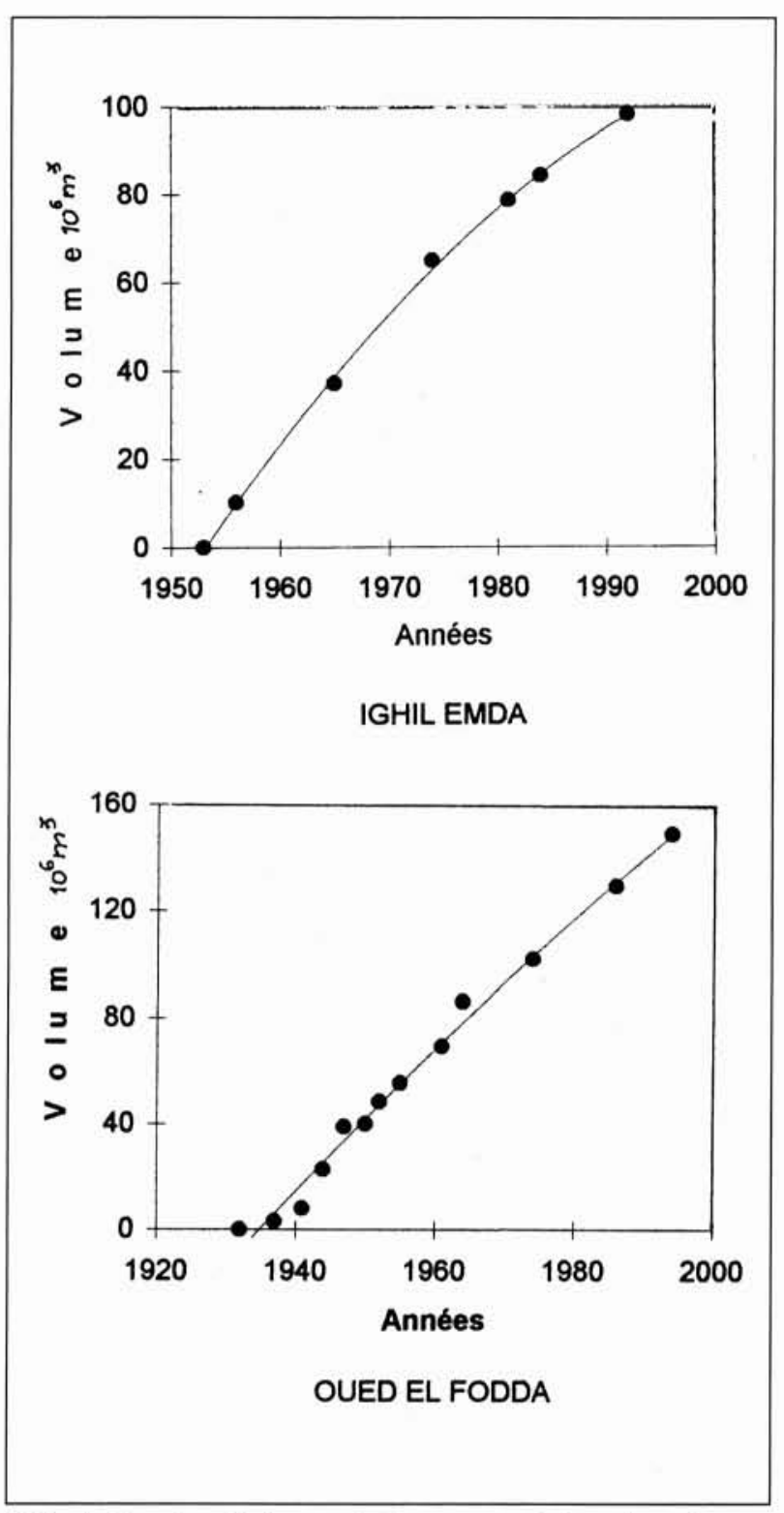

7. Evolution dans le temps de l'envasement dans les retenues de barrages en l'absence de soutirage des sédiments.

[3] Duquennois H.-1954- Sédimentation et soutirage des vases au barrage d'IGHIL EMDA. Gaz et électricité, compte rendu $\mathrm{N}^{\circ} 1$.

[4] HaDI T., ChaDi A. -1991- Quelques aspects sur l'envasement des retenues Algériennes. Communication colloque sur l'érosion des sols et l'envasement des barrages. Alger, 1-3 Décembre.

[5] Keller P., Bouchard J-P. -1989- Etude bibliographique de l'alluvionnement des retenues par des sédiments fins. Rapport interne EDF, E43/D46, juillet.

[6] Remini B., Avenard J-M., Kettab A. -1994- Evolution de l'envasement et évacuation des sédiments par les vannes de fond dans le barrage d'IGHIL EMDA. Communication au premier séminaire national sur l'hydraulique, Biskra, 5-7 décembre.

[7] Thevenin J. -1959- Etude de la sédimentation des barrages réservoirs en Algérie et moyens mis en œuvre pour préserver les capacités. Colloque international de LIEGE. Barrages et bassins de retenues. 